\title{
Experiences Gained during the Development of a Passive BSAR with GNSS Transmitters of Opportunity
}

\author{
M. Cherniakov, Rajesh Saini, Michael Antoniou, Rui Zuo, and Eleftherios Plakidis \\ Microwave Integrated Systems Laboratory (MISL), Department of Electronic, Electrical and Computer Engineering, \\ University of Birmingham, Edgbaston, Birmingham B15 2TT, UK
}

Correspondence should be addressed to M. Cherniakov, m.cherniakov@bham.ac.uk

Received 18 February 2008; Accepted 14 May 2008

Recommended by M. Greco

This paper presents an overview of the research conducted at University of Birmingham. It highlights and briefly discusses various systems parameters (e.g., resolution, power budget), problems (e.g., interference, heterodyne channel Doppler compensation), and signal processing algorithms (imaging, synchronization) required for successfully obtaining an image. The GLONASS satellite is used for experiential confirmation of the main results. All these results are presented and briefly discussed.

Copyright (c) 2008 M. Cherniakov et al. This is an open access article distributed under the Creative Commons Attribution License, which permits unrestricted use, distribution, and reproduction in any medium, provided the original work is properly cited.

\section{INTRODUCTION}

Synthetic aperture radar (SAR) works by collecting the echo returns from many pulses along the flight path and processing them into a single high-resolution radar image. In a monostatic SAR, the transmitter and receiver are on the same platform, whereas in a bistatic SAR (BSAR) the transmitter and receiver are separated by a distance that is comparable to the expected target distance. Examples of BSAR include airborne systems, where the transmitter and receiver are located on different aircrafts $[1,2]$. In a space-borne system, the transmitter and receiver are based on two or more satellites [3,4]. There is another subclass of bisatic SAR known as space-surface bistatic synthetic aperture radar (SS-BSAR). The SS-BSAR consists of a spaceborne transmitter and a receiver mounted on or near the earth's surface, see Figure 1 . The receiver could be airborne, mounted on a ground vehicle, onboard a ship, or even in a stationary position on the surface. For the stationary receiver, a nongeostationary satellite should be used to provide aperture synthesis.

The core of SS-BSAR systems is their asymmetric topology [5]. This is in contrast to a more usual bistatic SAR configuration, where the transmitter and the receiver are moving along collinear trajectories. The basic operation of SS-BSAR systems is much the same as the operation of other BSAR systems, the differences being introduced mainly as a consequence of the geometry employed.
University of Birmingham (UoB) has been carrying out active research in the area of SS-BSAR since 2003. The main aim of this research is to experimentally demonstrate the feasibility and performance of airborne SS-BSAR, utilising Global Navigation Satellite System (GNSS) as the transmitter of opportunity.

The goal of this paper is to give the reader an overview of the research conducted at the UoB. It highlights and briefly discusses various systems parameters (for resolution, power budget), problems (e.g., interference, motion compensation), and signal processing algorithms (imaging, synchronisation) required for successfully obtaining an image. The GLONASS satellite is used for experiential confirmation of the main results. All these results are presented and briefly discussed.

It should be noted that although we are using a particular satellite system, the structure and main parameters of the radar system are generic and could be used with different GNSS transmitters.

\section{RESOLUTION}

The GLONASS satellite was selected as the transmitter of opportunity for experimentation, despite its rather poor potential slant range resolution $(30 \mathrm{~m})$. This was due to the fact that it was the only one available at the time of experimentation. Another GNSS system (GPS) has a navigation signal with twice the spectral width, and hence $15 \mathrm{~m}$ 
TABLE 1: The main parameters of different GNSS signals.

\begin{tabular}{|c|c|c|c|c|c|c|c|}
\hline GNSS & \multicolumn{2}{|c|}{ GLONASS } & \multicolumn{3}{|c|}{ GPS } & \multicolumn{2}{|c|}{ Galileo } \\
\hline Channels (code) & $\mathrm{L} 1(\mathrm{P})$ & L2 (P) & $\mathrm{L} 1(\mathrm{P} / \mathrm{M})$ & L2 (P/M) & L5 & $\mathrm{E} 5 \mathrm{a} / \mathrm{b}$ & E5 $(\mathrm{E} 5 \mathrm{a}+\mathrm{E} 5 \mathrm{~b})$ \\
\hline Central frequency $(\mathrm{MHz})$ & $1602-1615$ & $1246-1257$ & 1575.4 & 1227.6 & 1176 & 1191.79 & 1191.795 \\
\hline Minimum power $(\mathrm{dBW})$ & -161 & -167 & $\begin{array}{c}-158 \\
-138\end{array}$ & $\begin{array}{l}\text { al beam) } \\
\text { t beam) }\end{array}$ & -154.9 & -157 & -154 \\
\hline Chip rate (Mcps) & 5.11 & 5.11 & $10.23 / 5.11$ & $10.23 / 5.11$ & 10.23 & 10.23 & 10.23 \\
\hline Aggregated bandwidth $(\mathrm{MHz})$ & - & - & $20-30$ & $20-30$ & - & - & $20-50$ \\
\hline Range resolution (m) & 30 & 30 & $5-8$ & $5-8$ & 15 & 15 & $3-8$ \\
\hline
\end{tabular}

TABle 2: Potential target detection range for different GNSS signals.

\begin{tabular}{lccccc}
\hline Transmitter & $\lambda(\mathrm{cm})$ & $V_{a}(\mathrm{~m} / \mathrm{s})$ & $\eta$ & $A_{e}\left(\mathrm{~m}^{2}\right)$ & $\Delta_{\mathrm{az}}(\mathrm{m})$ \\
\hline Galileo E5 & 25.2 & & & & \\
GPS L5 & 25.5 & 200 & 0.5 & 0.5 & 1 \\
GPS spot beam & 19 & & & 410 \\
GLONASS L1 & 18.8 & & & \\
\hline
\end{tabular}

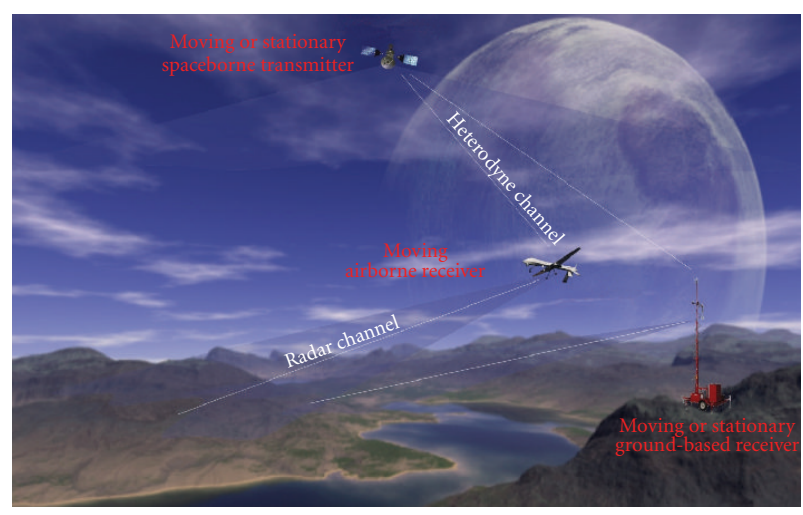

FIGURE 1: SS-BSAR concept for imaging.

potential range resolution, but this is a coded signal and can be used only with special authorisation. The most promising GNSS for the considered purposes are the EU Galileo system and the new proposed GPS signal structure [6]. Figure 2(a) shows the signal spectrum of Galileo E5 signal. Two signal components (E5a and E5b) in the E5 band are modulated as a single wideband signal generated following the AltBOC $(15,10)$ modulation. This wideband signal is centred at the frequency of $1191.795 \mathrm{MHz}$ and has a bandwidth of at least $50 \mathrm{MHz}$. Since the E5a and E5b signals transmit independent information from each other, it is possible in theory to develop a signal processing technique that combines the received E5a and E5b signals in such a way to give a resolution of 3-8 m. Similarly, the different signals (P-code and M-code) of the modernised GPS L1/L2 channel can be combined to achieve $20-30 \mathrm{MHz}$ bandwidth or 5-8 $\mathrm{m}$ range resolution. This makes GNSS (Galileo, GPS) based SS-BSAR a prospective candidate for many practical applications.

Table 1 summarises some GNSS signal parameters and their potential slant range resolution. The stated resolution is for a quasimonostatic configuration. However, for other configurations the resolution is dependent upon the geom- etry of the system, that is, satellite-receiver-target positions not relative to each other. The effect of the system's geometry on the resolution is comprehensively discussed in [7].

It should be noted that one of the key advantages of using a GNSS satellite compared to other satellites (e.g., Geostationary TV satellite) is that the user can choose the desired bistatic topology (low bistatic angle, i.e., negligible resolution loss). This is due to the fact that 4 to 10 GNSS satellites are simultaneously visible at any point on the earth. As a result, a particular satellite in the best (or at least suitable) position can be selected and there is no need for a very specific aircraft trajectory to allow the observation of an area. On the other hand, geostationary satellites are fundamentally positioned above the equator and this requires a specific aircraft trajectory for mapping a particular area and, in many or even most situations, a vital loss in ground resolution may take place.

\section{POWER BUDGET}

The receiving part of the SS-BSAR consists of two channels: the heterodyne channel (pointed directly towards the satellite) is used for synchronisation and the radar channel is used for receiving reflected signal from the target area. The power budget of the radar channel is a determining parameter for target detection, which is calculated for the time of aperture synthesis and considering only targets that have RCS independent of frequency and angle. For bistatic SAR, the expression for SNR after range and azimuth compression can be written as [8]

$$
\frac{S}{N}=\frac{\rho A_{e} \sigma \lambda \eta}{4 \pi R_{R} K T_{S} V_{a} \Delta_{\mathrm{az}}}
$$

where $\rho$ is power flux density of the transmitting signal in target area, $\sigma$ is the radar cross-section of the target, $\eta$ is a general loss factor, $R_{R}$ is the receiver-target range, $V_{a}$ is the receiver velocity, $\Delta_{\mathrm{az}}$ is the potential azimuth resolution.

Table 1 shows the minimum received signal power by $0 \mathrm{~dB}$ omnidirectional antenna on the ground for different 


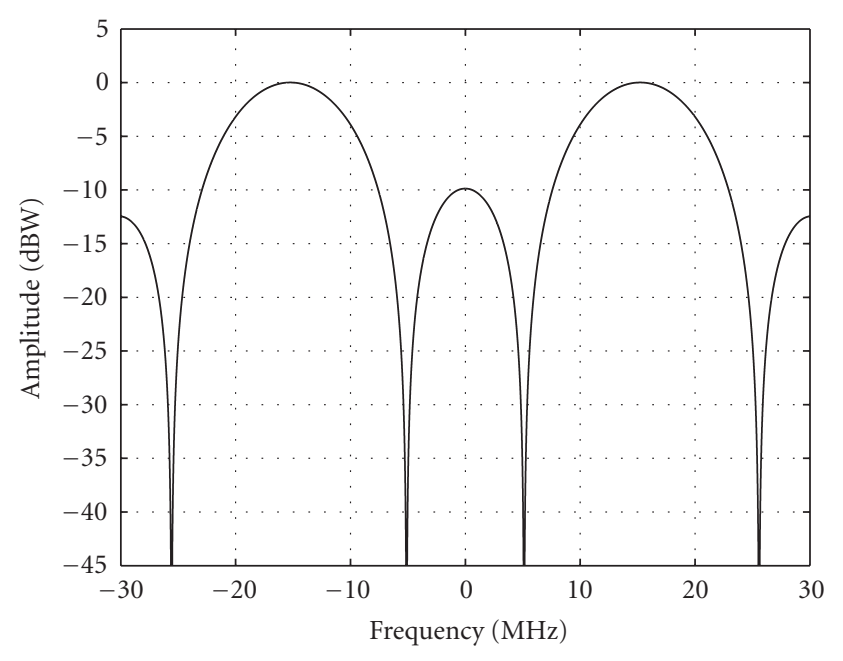

(a)

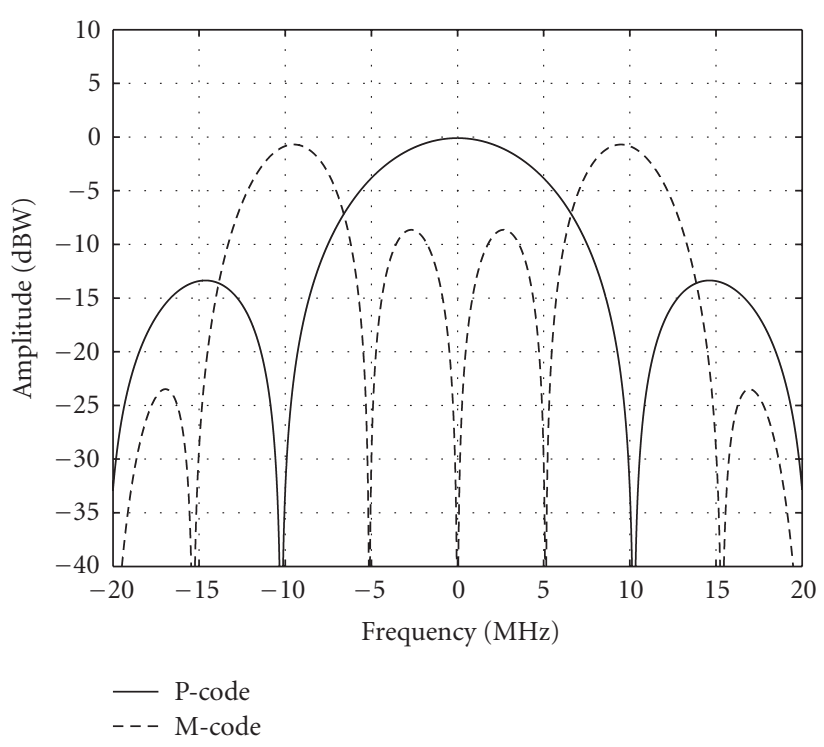

(b)

FIgUre 2: (a) Galileo E5 channel Spectrum, (b) GPS L1 or L2 channel Spectrum.

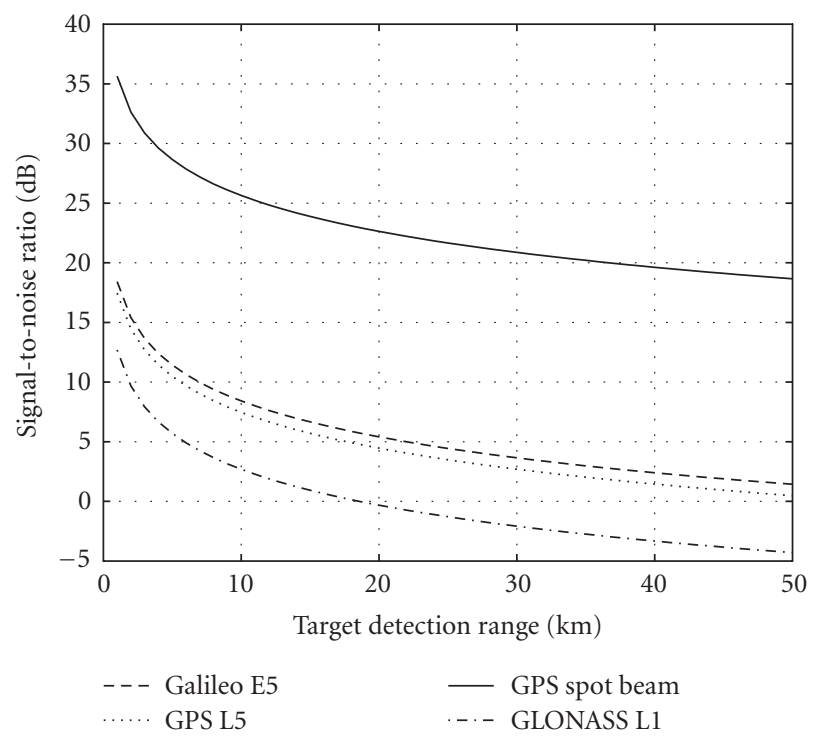

FIgURE 3: Detection range for $50 \mathrm{~m}^{2}$ RCS target.

GNSS signals. It can be noted that the Galileo E5 signal and new GPS L5 signal provide at least $6 \mathrm{~dB}$ more power compared to GLONASS L1 channel. In addition GPS after modernisation will broadcast the signal from a high-gain directional antenna, termed as spot beam, which generates $20 \mathrm{~dB}$ more power than its global beam and covers the ground area of $1200 \mathrm{~km} \times 1200 \mathrm{~km}$ that is enough for our application.

Using the parameters provided in Tables 1, 2, and (1), Figure 3 shows the potential range of SS-BSAR for different GNSS signals. If we consider $10-13 \mathrm{~dB}$ SNR as the radar detection threshold, targets with $50 \mathrm{~m}^{2}$ RCS can be detected at the range of approximately $5 \mathrm{~km}$ using Galileo E5 or GPS
L5 signal. By using GPS spot beam (available in 2013), the detection range can be extended to more than $50 \mathrm{~km}$ for target with $50 \mathrm{~m}^{2}$ RCS.

It also should be noted that all the above range calculations were done using the minimum power received for each signal. The minimum power level is received when the satellite is near the horizon and the maximum when the satellite is at an elevation of approximately $45^{\circ}$. On average, the difference between the minimum and maximum power level is around $6 \mathrm{~dB}$. Hence, one can expect four times more improvement in the maximum range when a satellite at an elevation of about $45^{\circ}$.

\section{INTERFERENCE}

Using GNSS as an illuminator in SS-BSAR presents a specific problem in signal detection. In addition to the desired target echo, a number of interference signals are present in the system. GNSS signals are modulated continuous waves and the desired reflected signal has to be detected against a continuous interference background. The first type of such interference is direct path interference (DPI), which is the signal received directly by the radar antenna from the illuminating satellite. The second type is adjacent channel interference (API) coming from another GNSS satellite operating in the same frequency band. These interference sources discussed in $[8,9]$ showed that the signal-to-interference ratio (SIR) is high enough to detect the desired signal. It was shown that the interference and the desired signal are essentially the same process, and at the output of the matched filter the interference level is specified by the cross-correlation side-lobes. In addition, the SAR algorithm itself acts as a spatial filter to these interference sources. The fact that the interference signals are coherent to the desired signal introduces some low-level false targets, 


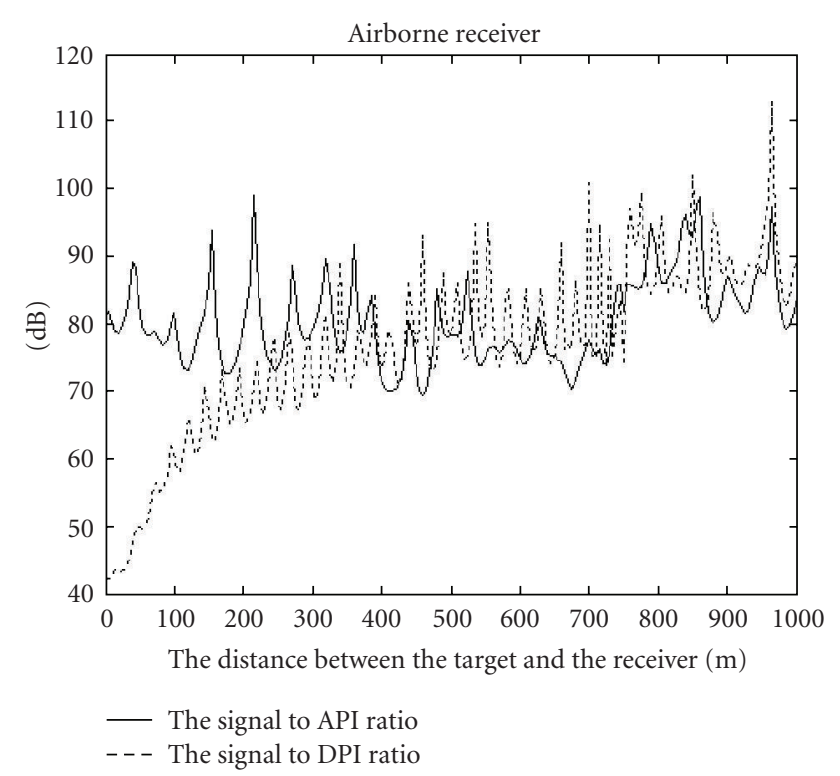

Figure 4: Signal-to-interference ratio [9] (zoomed).

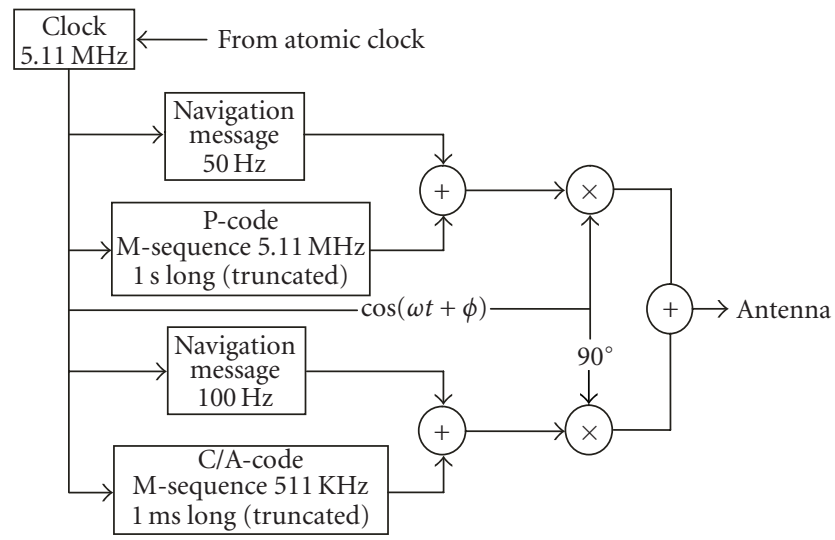

FIgURE 5: Simplified block diagram of the L1 GLONASS signal structure.

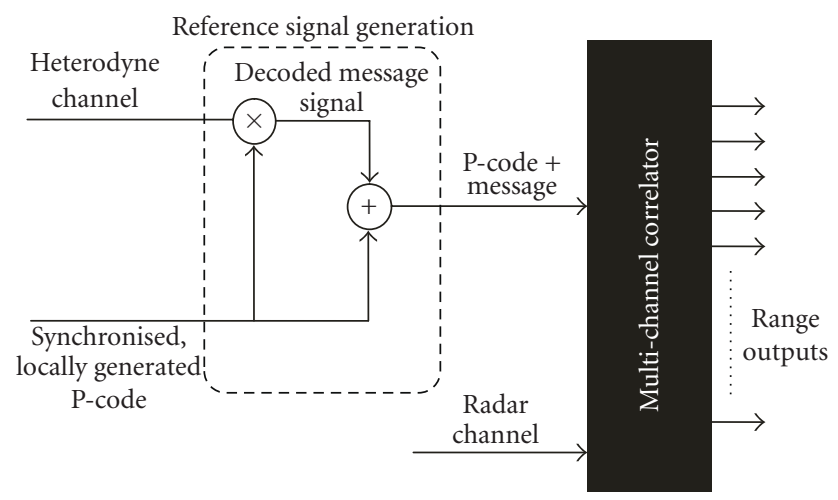

๑ Module-2 addition

FIGURE 6: Range compression algorithm. but these false targets will be outside the observation area. Figure 4 shows the SIR evaluated for a practical situation [9].

\section{GLONASS SIGNAL STRUCTURE AND SYNCHRONISATION}

Figure 5 shows a simplified block diagram of the structure of GLONASS signals transmitted in the L1 frequency band [10]. The C/A and P-code signals are in phase quadrature. The C/A code rate is $511 \mathrm{KHz}$ and the code period is 1 millisecond. The $\mathrm{C} / \mathrm{A}$ code sequence is added $(\bmod 2)$ to a $100 \mathrm{~Hz}$ navigation message. The $\mathrm{P}$-code has a chip rate of $5.11 \mathrm{MHz}$ and is a truncated $\mathrm{M}$-sequence repeated every 1 second. The navigation message on the P-code has a clock rate of $50 \mathrm{~Hz}$.

Mathematically the transmitted signal can be written as

$$
Y=A_{p} P(t) D_{p}(t) \cos \left(\omega_{c} t+\phi\right)+A_{C} C(t) D_{c}(t) \cos \left(\omega_{c} t+\phi\right),
$$

where $Y$ is the signal at $\mathrm{L} 1$ frequency, $A_{p}(t)$ is the amplitude of the P-code, $P(t)$ represents the polarity $( \pm 1)$ of the P-code, $D_{p}(t)$ is the binary $( \pm 1) \mathrm{P}$-code navigation message, $\omega_{c}$ is the L1 frequency, $\phi$ is the initial phase, $A_{c}(t)$ is the amplitude of the C/A code, $C(t)$ represents the polarity $( \pm 1)$ of the C/A code, $D_{c}(t)$ is the binary $( \pm 1) \mathrm{C} / \mathrm{A}$ navigation message.

The P-code will be used for the purpose of imaging, as it provides a reasonable range resolution of about $30 \mathrm{~m}$ (quasimonostatic case) and is no longer encrypted. Usually, in a radar signal processor, the range compression consists of a correlation of the radar channel signal with the heterodyne channel signal delayed for each range resolution cell in the multichannel correlator. In our previous publication [7], it was demonstrated that for GLONASS signal it is not possible to directly correlate the heterodyne channel with the radar channel. For a quick reminder, we briefly discuss the reason below.

The signal received from the GLONASS satellite is a superposition of the C/A code and P-code signals, the spectra of which overlap; the P-code (5.11 MHz bandwidth) is used for the purpose of imaging, as mentioned earlier. If the heterodyne channel is directly correlated with the radar channel, the P-code will be masked by the C/A code at the output of the correlator. The bandwidth of the C/A code is only one-tenth that of the P-code but even if the C/A code of the desired satellite signal is filtered out in the heterodyne channel, the signal correlation properties are degraded by the C/A codes of interfering satellites (It should be noted that this is a peculiarity of only GLONASS signal. In GPS, $\mathrm{C} / \mathrm{A}$ codes are transmitted on the same carrier frequencies by different satellites. Therefore, the C/A code can be filtered out for all the satellites in the heterodyne channel). It was also demonstrated that, if the radar channel signal is correlated with a locally generated signal containing only the P-code, the effect of the C/A code could be suppressed. However, this technique needs navigation message decoding which, in turn, requires P-code synchronisation. Figure 6 shows the principles of the proposed range compression algorithm.

The synchronisation compromises of tracking the satellite in delay, Doppler, and phase in order to fully decode the 


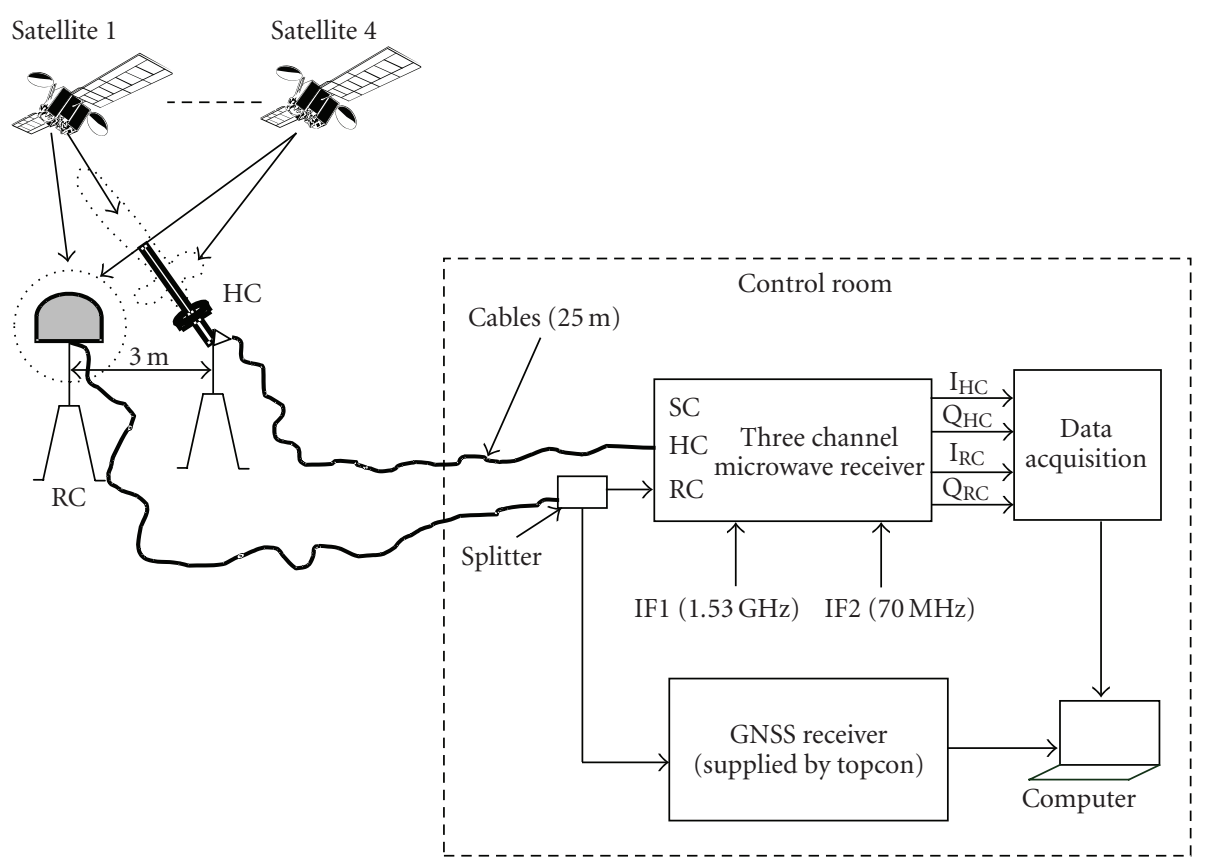

FIGURE 7: Experimental setup for confirming synchronisation.

navigation message. The "block adjustment of synchronising signal (BASS)" algorithm for Doppler extraction was used. This algorithm is used in software GPS receivers and is comprehensively discussed in [11]. For delay tracking and algorithm based on conventional delayed-locked loop was used [11]. It should be noted that a detailed discussion of the synchronisation algorithms is outside the scope of this paper. Here, we only present the experiment results.

Figure 7 shows the experimental setup for collecting data from the satellite to verify the range compression and synchronisation algorithms. The signal from the satellite was directly received on two channels (radar and heterodyne channels). The radar channel antenna was a stationary omnidirectional antenna (6 dBi gain) and was separated by some $3 \mathrm{~m}$ from the heterodyne channel antenna. This topology corresponds to a target at "zero range." For the heterodyne channel, a directional spiral antenna with a gain of $16 \mathrm{dBi}$ was pointed towards the satellite. A satellite transmitting on channel $10(1607.625 \mathrm{MHz})$ was used.

Figures $8(\mathrm{a})$ and $8(\mathrm{~b})$ shows the estimated frequency and delay variation of the satellite. From Figure 8(b), it is seen that the rate of frequency variation is around $0.4 \mathrm{~Hz} / \mathrm{sec}$. Figure 8(c) shows the decoded message signal from the P-code after phase tracking. These results confirm the proper functioning of our algorithm. Figure $8(d)$ represents the correlation obtained from the proposed range compression algorithm shown in Figure 6. It is seen that the algorithm gives a good correlation and confirms our computer modelling result. As expected, the width (first null) of the main lobe is 0.2 microsecond and the RMS sidelobe level of $-60 \mathrm{~dB}$ for 45 seconds integration time.

\section{HETERODYNE CHANNEL DOPPLER COMPENSATION}

Figure 9 shows the experimental setups used to verify the imaging algorithm (discussed in the next section). The first setup consisted of stationary heterodyne channel and moving radar channel (for antenna synthesis). The second experiment represents a more practical scenario, where both the heterodyne and radar antennas are moving. It is obvious that the only difference between the two setups is the presence of extra Doppler shift in the moving heterodyne channel. This Doppler shift can be large enough to introduce a complete mismatch between the heterodyne channel and the radar channel. Hence, one needs to compensate the relative motion between transmitter and receiver.

First of all, we consider an instantaneous bistatic triangle formed by the stationary heterodyne channel, shown in Figure 10(a). The three arms of the bistatic triangle are transmitter-to-receiver path (represented by $B$ ), transmitterto-target path $\left(R_{T}\right)$, and target-to-receiver $\left(R_{R}\right)$. Also, the bistatic angle is denoted by $\beta$, the transmitter elevation angle by $\varphi$, and the angle between $B$ and $R_{T}$ by $\theta_{T r}$. For our experimentation with flight imitator we considered targets at short ranges (maximum target range $600 \mathrm{~m}$ ). The typical transmitter-to-receiver range for GLONASS is $\sim 20000 \mathrm{~km}$. Therefore, the angular separation, $\theta_{T r}$, is negligible. Hence, for a stationary heterodyne channel, the Doppler shifts due to satellite motion $\left(F_{S}\right)$ in the two paths are similar. The residual Doppler variation after range compression is present only due to receiver motion relative to target at $R_{R}$. This residual Doppler variation in the radar channel forms the azimuth signature of a target. In the imaging algorithm, an appropriate azimuth compression filter is designed for each range bin accordingly. 


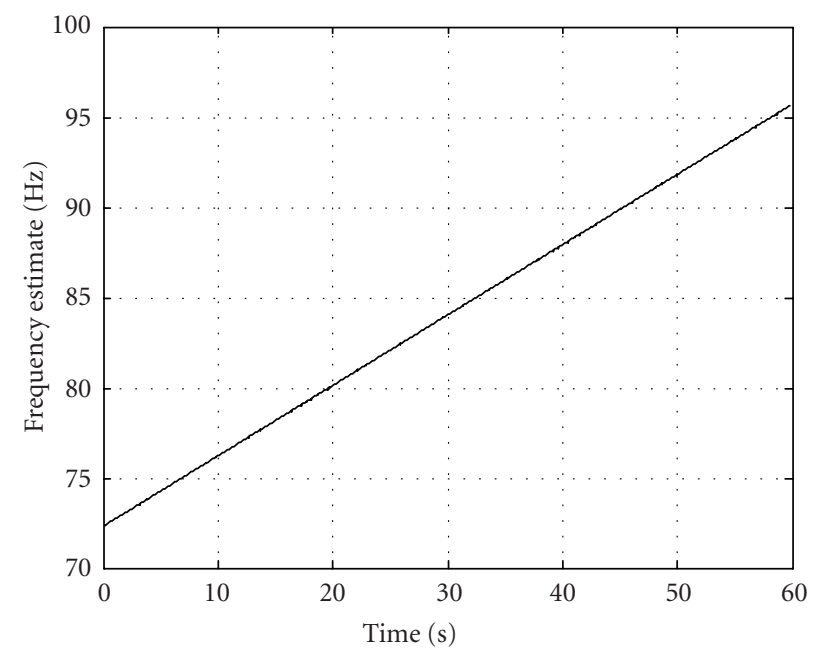

(a)
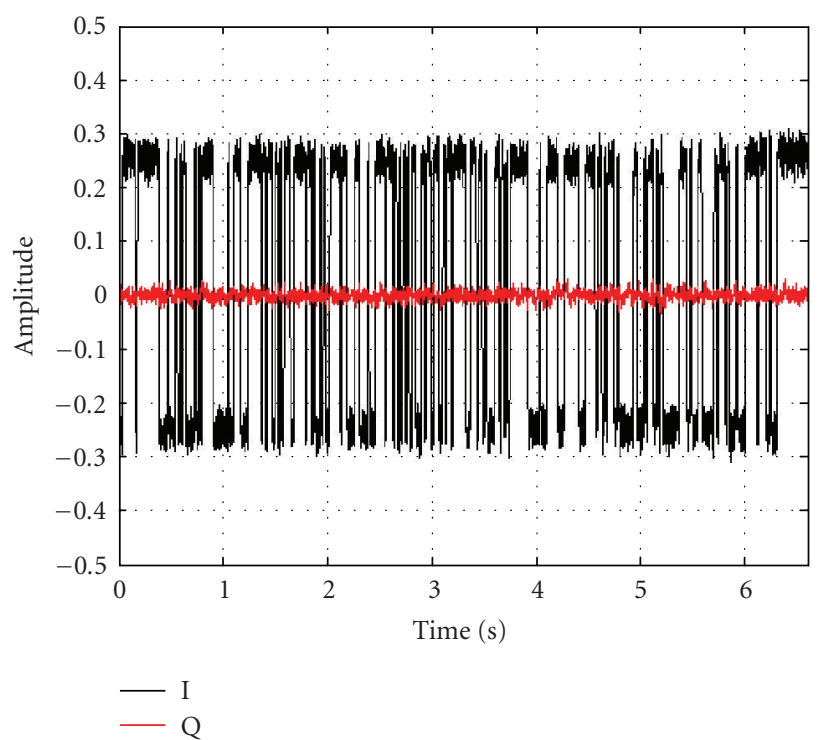

(c)

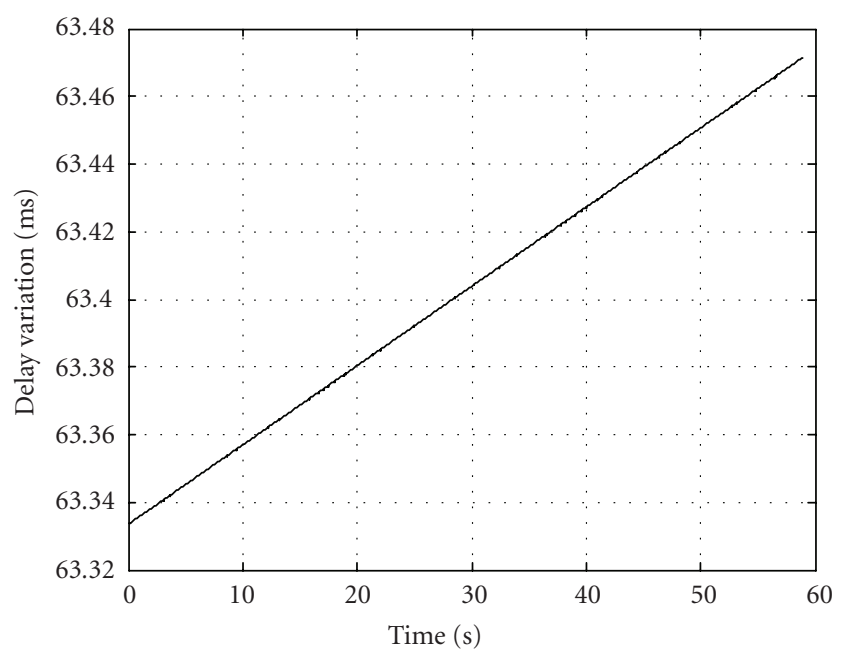

(b)

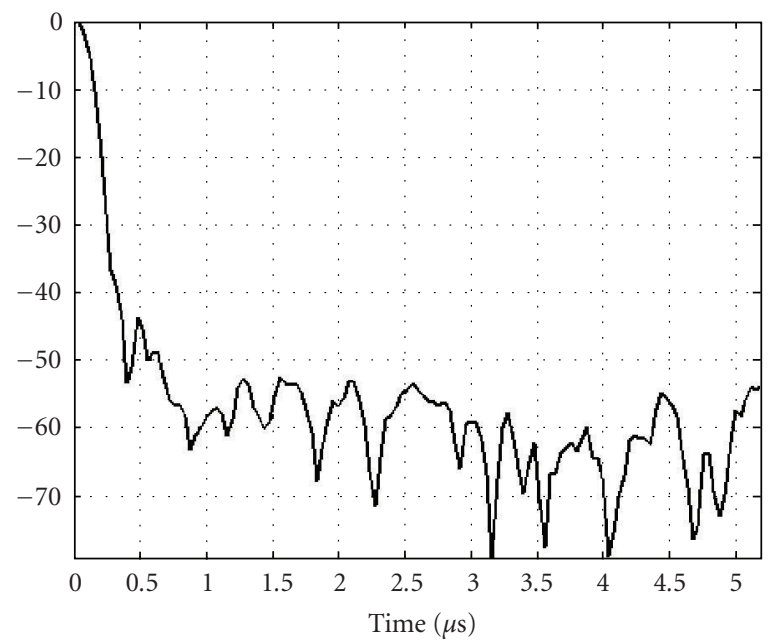

(d)

Figure 8: (a) Estimated frequency, (b) estimated delay variation (c), decoded navigation message, and (d) output of range compression algorithm.

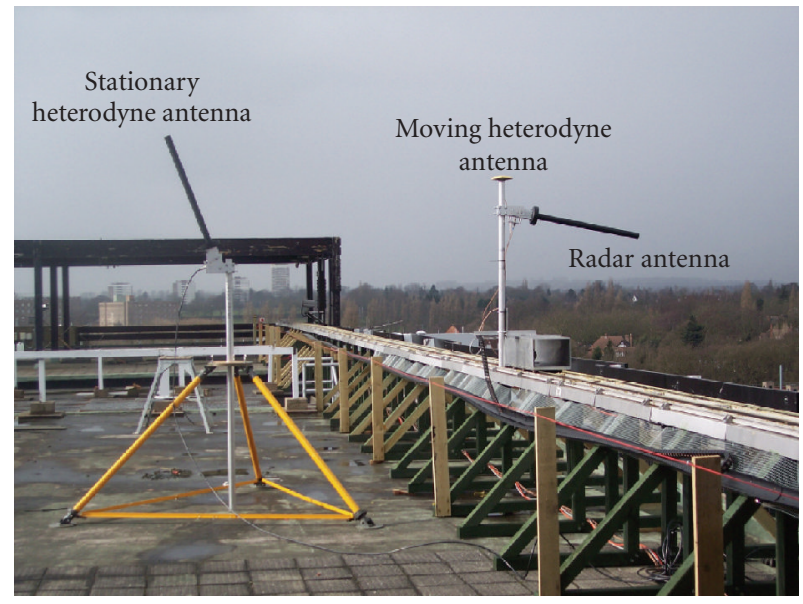

Figure 9: Experimental setups.
In a practical scenario, the heterodyne channel will be in motion as it is mounted on a real aircraft. Figure 10(b) shows the bistatic triangle for moving heterodyne channel. The only difference compared to the stationary case is the additional presence of Doppler variation $\left(F_{\mathrm{RS}}\right)$ due to receiver motion relative to the satellite in the heterodyne channel. It is clear from the figure that the residual Doppler variation is $F_{\mathrm{RS}}+F_{R}$. The question whether $F_{\mathrm{RS}}$ can be ignored when designing the azimuth filter is discussed below.

For 20 seconds integration time, the maximum tolerable frequency difference between the designed azimuth signature and the actual received signal is $\sim 0.003 \mathrm{~Hz}$ (Considering $\pi / 8$ phase difference over the integration time.). The value of $F_{\mathrm{RS}}$ can range from $10-100 \mathrm{~Hz}$ for an aircraft moving with a velocity of $30 \mathrm{~m} / \mathrm{sec}$. Hence, $F_{\mathrm{RS}}$ should be estimated and incorporated in the azimuth filter. It should be noted that this problem is not faced in a monostatic SAR as the transmitter 


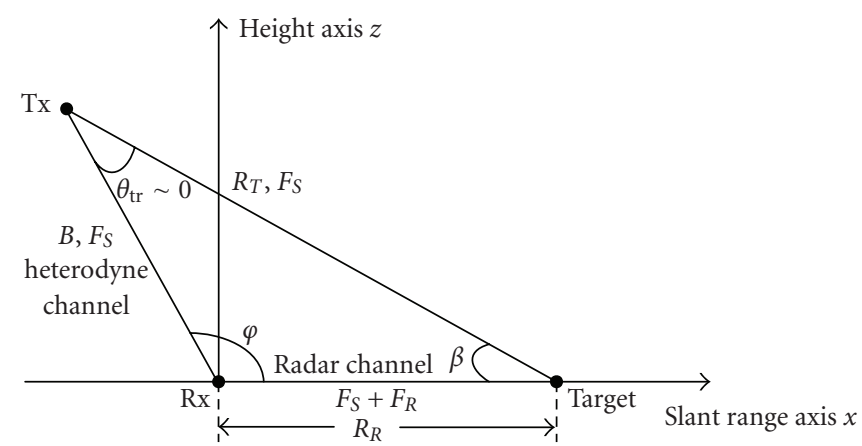

$F_{S}:$ Doppler due to satellite motion $F_{R}$ : Doppler due to moving receiver $B$ : Transmitter-to-heterodyne range $R_{T}$ : Transmitter-to-target range

(a)

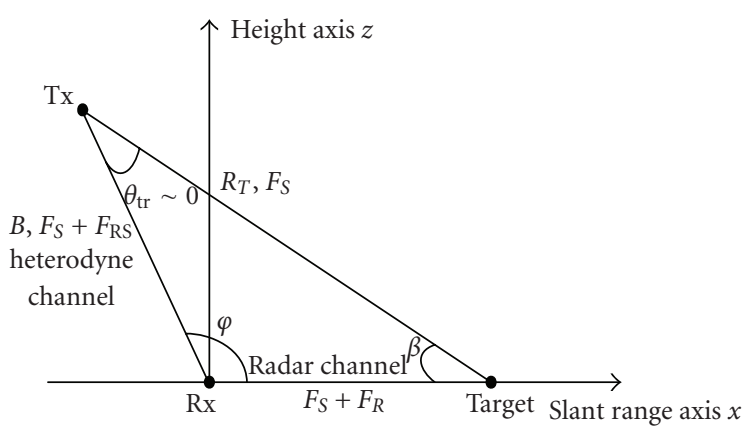

$F_{\mathrm{RS}}$ : Doppler due to receiver motion relative to the satellite

FIGURE 10: Bistatic triangle for (a) stationary heterodyne and (b) moving heterodyne.

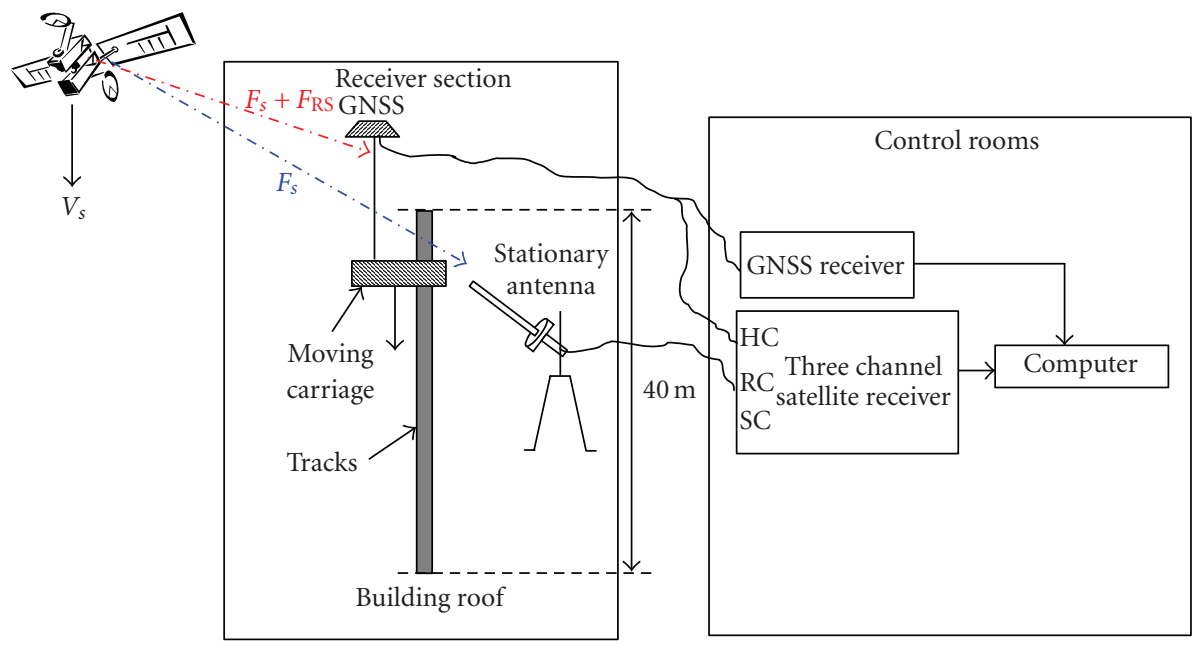

FIGURE 11: Experimental setup to calculate $F_{\mathrm{RS}}$.

and receiver are colocated. The method we used to estimate $F_{\mathrm{RS}}$ is explained in the following section with the aid of an experimental setup.

\subsection{Experimental setup to estimate $F_{\mathrm{RS}}$ and to verify moving heterodyne synchronisation}

Figure 11 shows the experimental setup for collecting data from the GLONASS satellite to estimate $F_{\mathrm{RS}}$ and to verify the moving heterodyne synchronisation (We used GLONASS satellite as GALILEO was not available at the time of experimentation. However, this is a generic problem and the analysis can be extended to any transmitter used in SSBSAR.). The signal from the satellite was directly received on two channels (moving and stationary). The moving channel antenna was an omnidirectional antenna and was separated by some $3 \mathrm{~m}$ from the stationary channel antenna. The moving channel corresponds to a moving heterodyne channel and we assume the stationary antenna to be a target at "zero range." In this experiment, we are ignoring the radar channel as the sole aim of the experimentation is to verify whether we can correctly estimate the $F_{\mathrm{RS}}$.

The two channels were simultaneously fed to a microwave receiver that down-converts the received signal through appropriate filtering and amplification. The base-band signal was then stored on a PC for off-line processing. The output of the moving channel is also fed to a second "GNSS receiver." This receiver stores the navigation message of the GPS/GLONASS satellite and receiver observation data. An algorithm was developed that extracts the satellite and the moving antenna positions from the stored data. From this information we calculated the $F_{\mathrm{RS}}$, that is, Doppler variation due to receiver motion relative to the satellite.

To test that whether we have correctly estimated the $F_{\mathrm{RS}}$, first of all we correlated the moving and the stationary channel without compensating $F_{\mathrm{RS}}$ from the moving channel. Due to presence of Doppler difference $\left(F_{\mathrm{RS}}\right)$ between the two channels, one can expect degradation of correlation 
function and rise in sidelobe level. Figure 12(a) shows the degradation of correlation function and Figure 12(c) shows a rise in the sidelobe level (dotted line). Figure 12(b) shows the correlations of the two channels after we have removed the $F_{\mathrm{RS}}$ from the moving channel. It is seen that after removing the residual Doppler we obtain a good correlation function. As expected, the width (first null) of the main lobe is 0.2 microsecond and the RMS sidelobe level of $-64 \mathrm{~dB}$ for 35 seconds integration time (Figure 12(c)). Hence, our estimate of $F_{\mathrm{RS}}$ is correct.

\section{SS-BSAR IMAGING}

In this section, a conceptual description of the algorithm used for SS-BSAR image formation will be provided, along with some experimental results. A specific configuration is assumed, where the transmitter is a GNSS satellite and the receiver is airborne. A full description of the algorithm can be found in $[12,13]$, along with detailed descriptions of algorithms designed for other SS-BSAR configurations. Results of our research in this area can also be found in [14-17].

\subsection{Algorithm description}

Before proceeding into an explanation of the algorithm, it is perhaps useful to state problems associated with SSBSAR image formation first. Traditional monostatic SAR algorithms exploit similarities in echo returns from different targets within the illuminated scene in order to reduce the signal processing load. These similarities are usually present in the range or azimuth target signatures and allow efficient processing in the frequency domain. In BSAR, and particularly SS-BSAR, these similarities do not exist. Moreover, the exact relationship between the azimuth-time signature (a chirp signal) of a target and its Doppler frequency spectrum is yet to be calculated analytically (at least without some approximation). These features are impediments to the derivation of efficient and accurate image formation algorithms operating in the frequency domain.

A block diagram of the generalised SS-BSAR image formation algorithm is shown in Figure 13. It may be noticed that it is a modification of the standard Range-Doppler algorithm, widely used in monostatic SAR image formation, and thoroughly described in [18].

The first step in the algorithm is to equalize the Range and Doppler histories of targets residing at the same range from the receiver. This is achieved by applying a single correction function to the SS-BSAR data, which removes the time delay and associated phase due to the transmitterto-receiver range at each azimuth-time instant. The correction function is integrated with the range compression step, hence the term "modified range compression." The modified range compression is performed in the rangefrequency, azimuth-time domain. At the output of this step, the Range and Doppler histories of targets at the same range, but different cross-range becomes very similar for a wide range of possible geometries. Since the transmitterto-target and receiver-to-target ranges can normally be

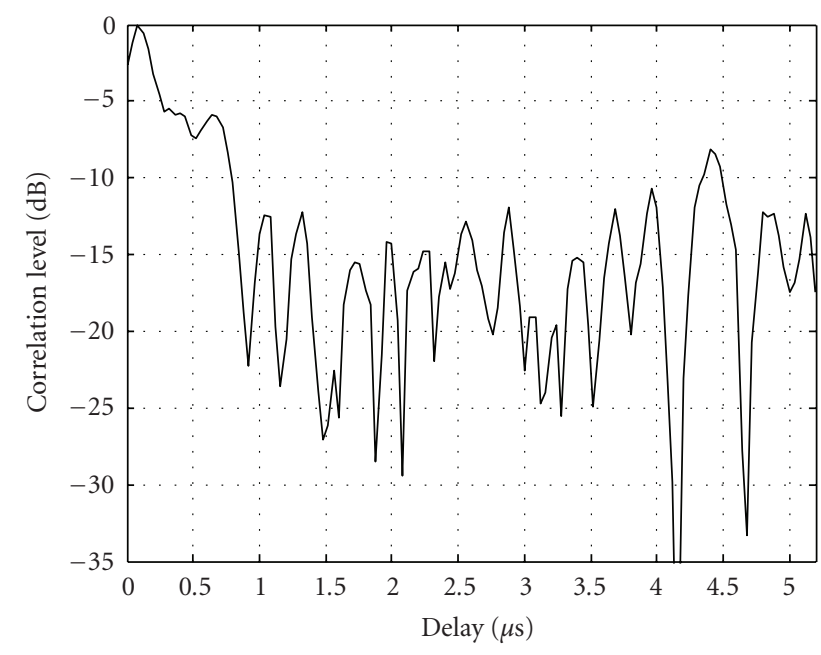

(a)

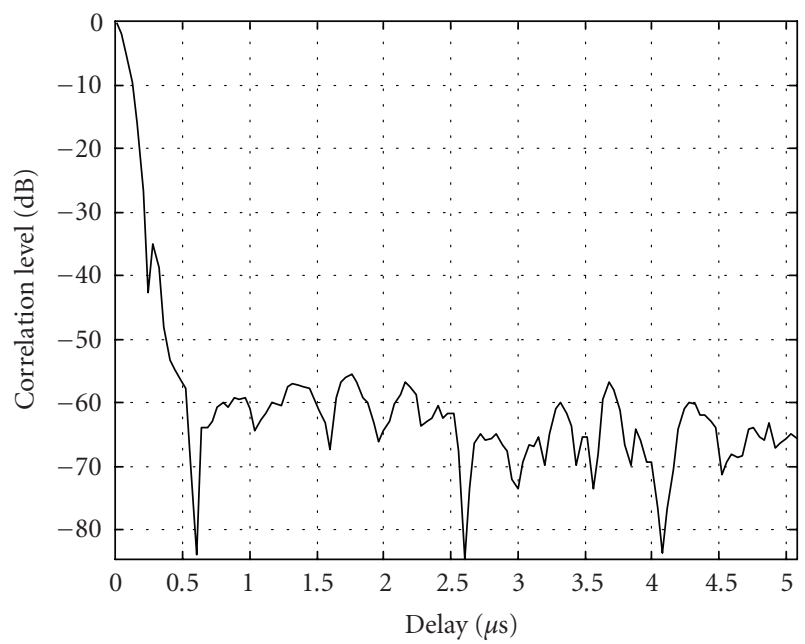

(b)

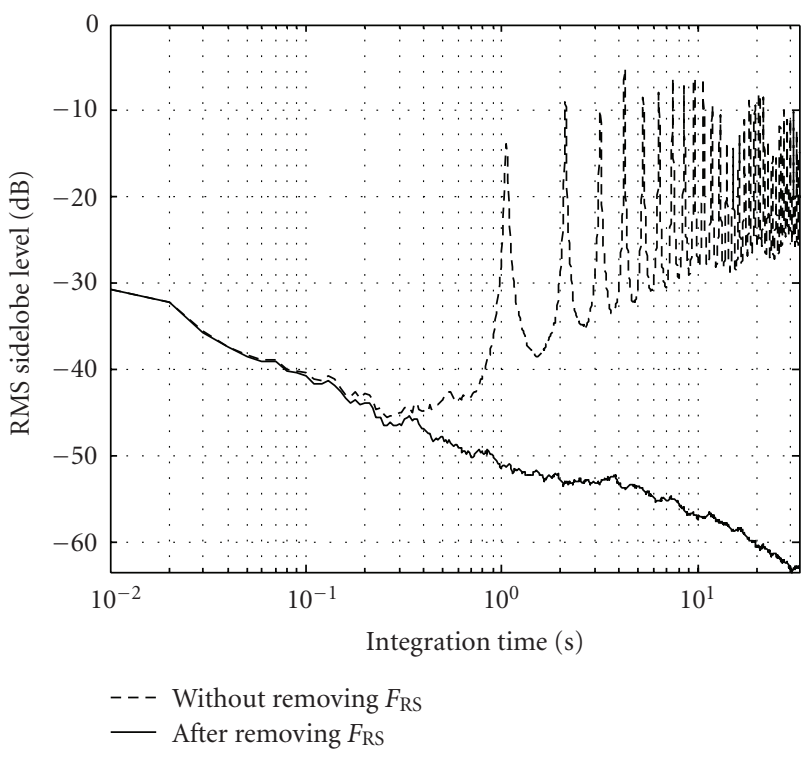

(c)

FIGURE 12: (a) Correlation without removing $F_{\mathrm{RS}}$, (b) correlation after removing $F_{\mathrm{RS}}$, and (c) RMS sidelobe level versus integration time (log scale). 


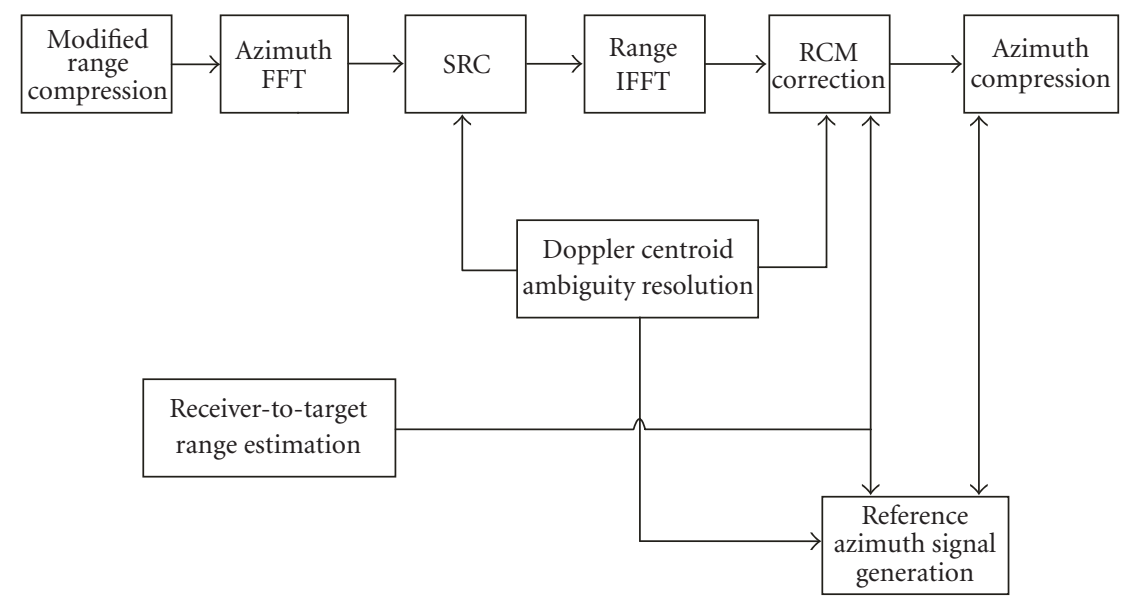

FIGURE 13: Block diagram of SS-BSAR image formation algorithm.

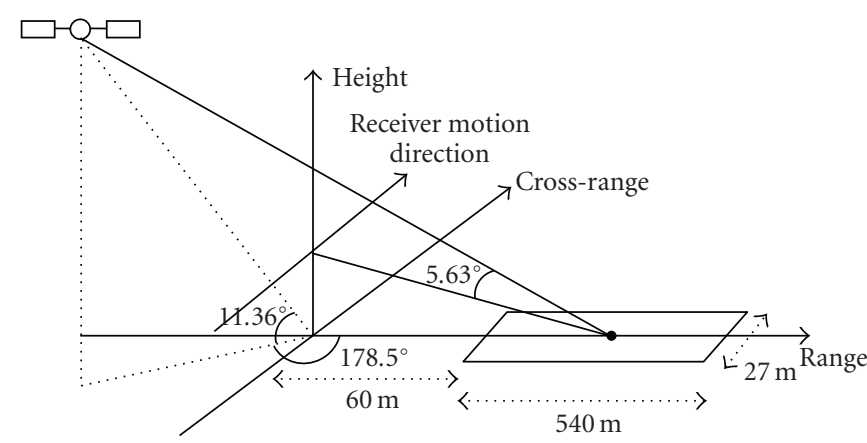

FIGURE 14: SS-BSAR experiment geometry.

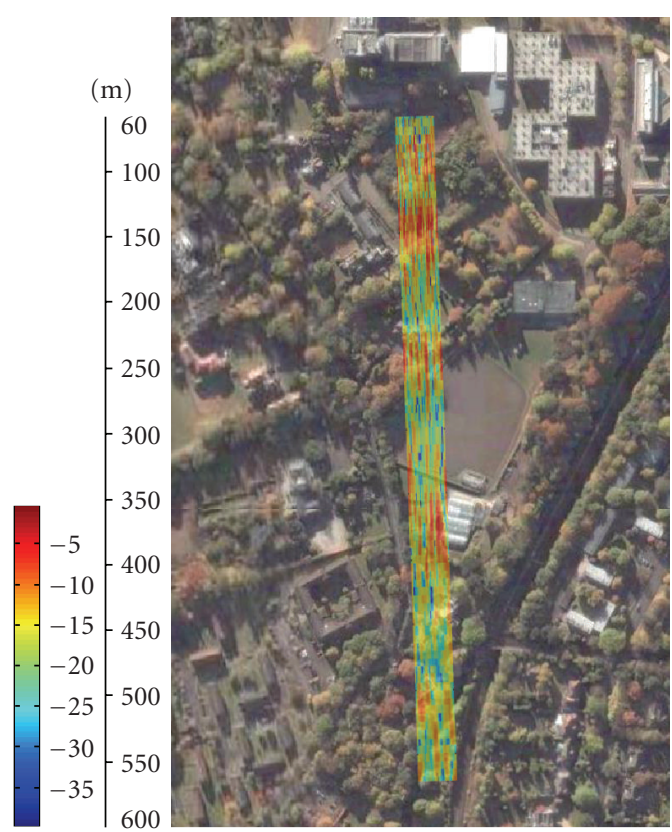

(a)

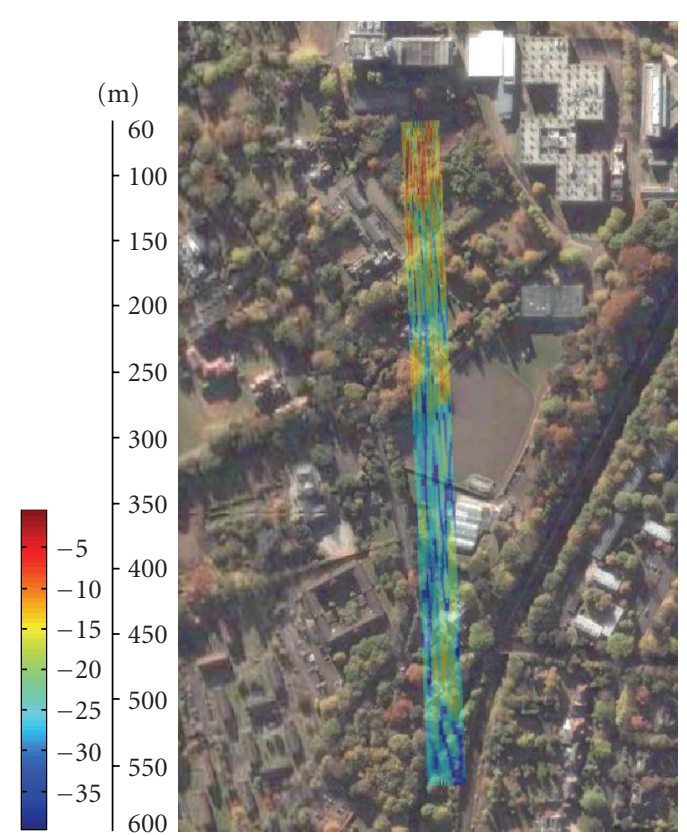

(b)

FIGURE 15: SS-BSAR image for (a) stationary heterodyne and (b) moving heterodyne. 
TABLE 3: Experimental parameters.

\begin{tabular}{lcc}
\hline Parameter & Stationary heterodyne & Moving heterodyne \\
\hline GLONASS satellite & COSMOS 2394 & COSMOS 2418 \\
Carrier frequency (MHz) & 1603.125 & 1603.125 \\
Signal bandwidth (MHz) & 5.11 & 5.11 \\
Satellite's azimuth angle (degree) & 178.49 & 182 \\
Satellite's elevation angle (degree) & 11.36 & 38 \\
Bistatic angle (degree) & 5.63 & 38 \\
Receiver velocity (m/sec) & 0.6 & 0.6 \\
Receiver's height above the ground (m) & $\sim 25$ & $\sim 25$ \\
Receiver's aperture length (m) & 27 & 27 \\
Integration time (s) & 45 & 45 \\
\hline
\end{tabular}

approximated using second-order Taylor series expansions in SS-BSAR, it is also possible to derive signal expressions in the frequency domain. Therefore, use of a modification of the Range-Doppler algorithm is a convenient method to form the image of an observation area. A secondary range compression (SRC) is performed in the two-dimensional frequency domain to compensate for the cross-coupling between the range and azimuth frequencies. Before this operation is executed, the Doppler ambiguity is resolved (i.e., because the target azimuth signature could contain a large Doppler centroid outside the range of sampled azimuth frequencies). Range cell migration (RCM) is corrected in the range-time, azimuth-frequency (or Range, Doppler) domain, after RCM components due to the receiver motion and residual RCM after the modified range compression are calculated. For this operation, it is proposed to estimate the receiver-to-target range from the range sum (the difference between the total range history and the transmitter-to-receiver range history) in order to identify the individual RCM components mentioned above. Finally, azimuth compression is performed in the range, Doppler domain.

\subsection{Experimental results}

In this section, we present the SS-BSAR images obtained by applying the proposed algorithm on experimental data collected from the experimental setups shown in Figure 7. A detailed description of our experimental hardware (including the flight imitator) can be found in [19]. The experimental parameters are shown in Table 3 . The geometry of the first experiment is shown in Figure 14.

Figure 15(a) shows the SS-BSAR image obtained for a stationary heterodyne channel $\left(5.63^{\circ}\right.$ bistatic angle). The image obtained from the observation area is superimposed on a satellite photograph of the area. The color-scale is in $\mathrm{dB}$, where $0 \mathrm{~dB}$ represents the highest processed echo intensity. It is important to note that the heterodyne channel was stationary, so there was no need for the motion compensation described in Section 6. However, the signal synchronisation described in Section 5 was required. Estimates of the satellite's instantaneous Doppler frequency and time delay with respect to the heterodyne antenna (provided by the synchronisation algorithm) were used for the modified range compression. We can see from Figure 15(a) that the intensity variation in the SS-BSAR image obtained corresponds to the variation in the strength of echoes from the observed area. More analysis and verification on the obtained image can be found in [20].

Figure 15(b) shows the SS-BSAR image obtained for moving heterodyne channel $\left(38^{\circ}\right.$ bistatic angle). Therefore, all the algorithms described in previous sections (synchronisation, motion compensation, and imaging) were applied to the acquired data. The experimental parameters are also included in Table 3.

Comparing Figures 15(a) with 15(b) shows high level of similarity. Strong reflections at the near field are observed in both images. Also, targets at the range of $240 \mathrm{~m}, 350 \mathrm{~m}, 500 \mathrm{~m}$ are visible in both images. Figures 16(b) and 16(c) show the strong reflections from the building at $240 \mathrm{~m}$ in both images. The dynamic range is almost same in both images, although the signal strength in second image is lower than the first one. This is due to possible bistatic RCS reduction.

Figure 17 shows cross-sections of the building shown in Figure 16(a), taken along the azimuth direction for each of the SS-BSAR images. It is observed that even though the physical characteristics of the building have not changed, image reflectivity has changed. This can be because the imaging geometry between the two acquisitions is different.

\section{CONCLUSIONS}

This paper gave an overview of the research carried out by University of Birmingham in the area of SS-BSAR, utilising microwave emissions from GNSS transmitters as the ranging signal. GLONASS satellite was used for experimentation.

In the paper, different GNSS transmitters (GPS, GLONASS, and GALILEO) were compared on the basis of resolution and power budget. It was highlighted that GALILEO and the new GPS satellite are the most prospective candidate for SS-BSAR. GALILEO E5 signal has potential range resolution of $3-8 \mathrm{~m}$ and can detect targets with $50 \mathrm{~m}^{2} \mathrm{RCS}$ at a range of approximately $5 \mathrm{~km}$. On the other hand, the new GPS signal can also provide the same range resolution, but has 10 times more detection range compared to GALILEO. 


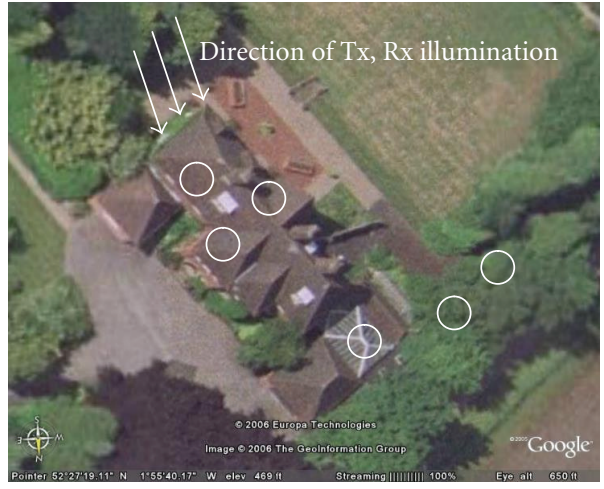

(a)

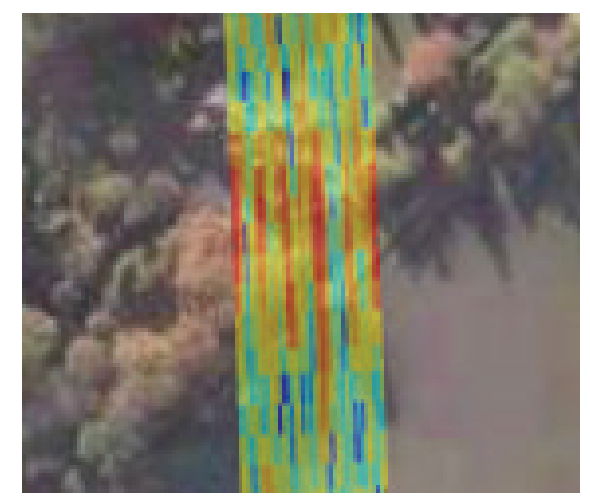

(b)

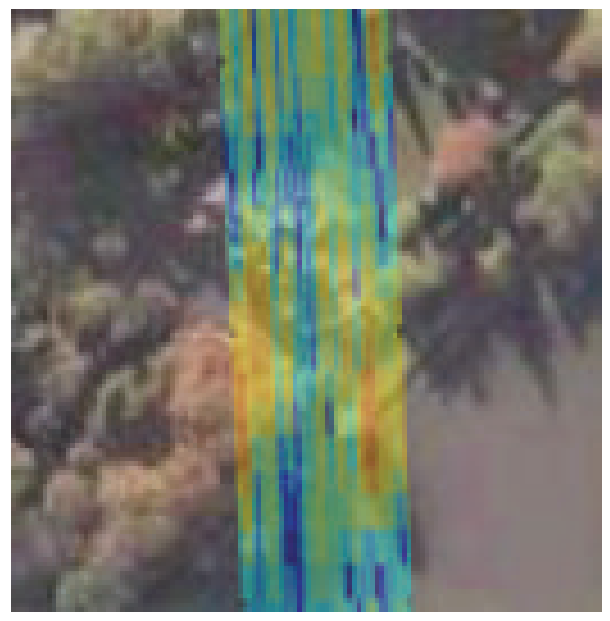

(c)

FIgUre 16: (a) Aerial photo of building at $240 \mathrm{~m}$, (b) enlargement of image area around the building at $240 \mathrm{~m}$ for $10^{\circ}$ bistatic angle, and (c) enlargement of image area around the building at $240 \mathrm{~m}$ for $40^{\circ}$ bistatic angle.

Some of the practical problems (such as interference, signal structure, synchronisation) were also briefly discussed. One of the important problems was moving heterodyne channel. It was discussed that the motion of the heterodyne channel introduces a Doppler shift. This Doppler shift needs to be estimated and incorporated in the azimuth compression filter design. A method to estimate this Doppler shift was described and experimentally verified.

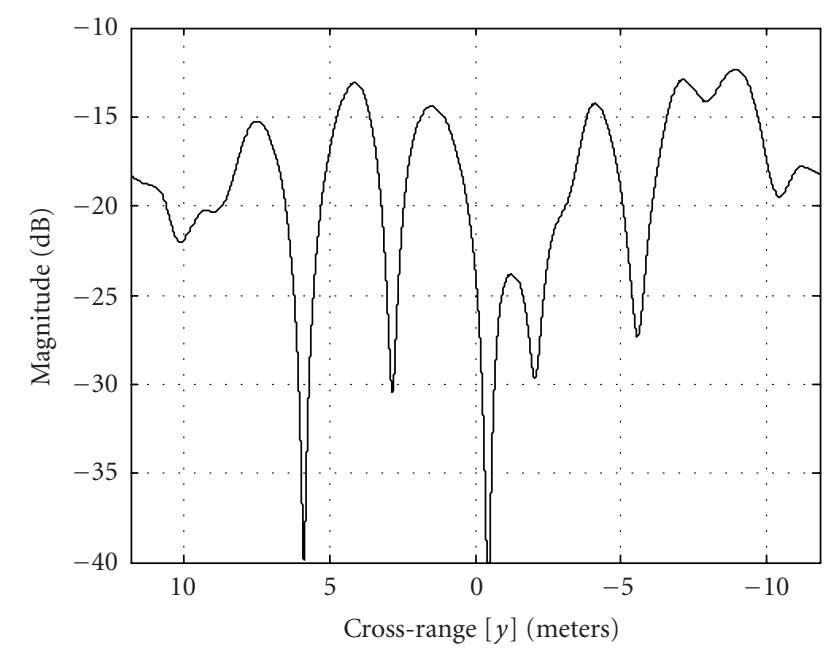

(a)

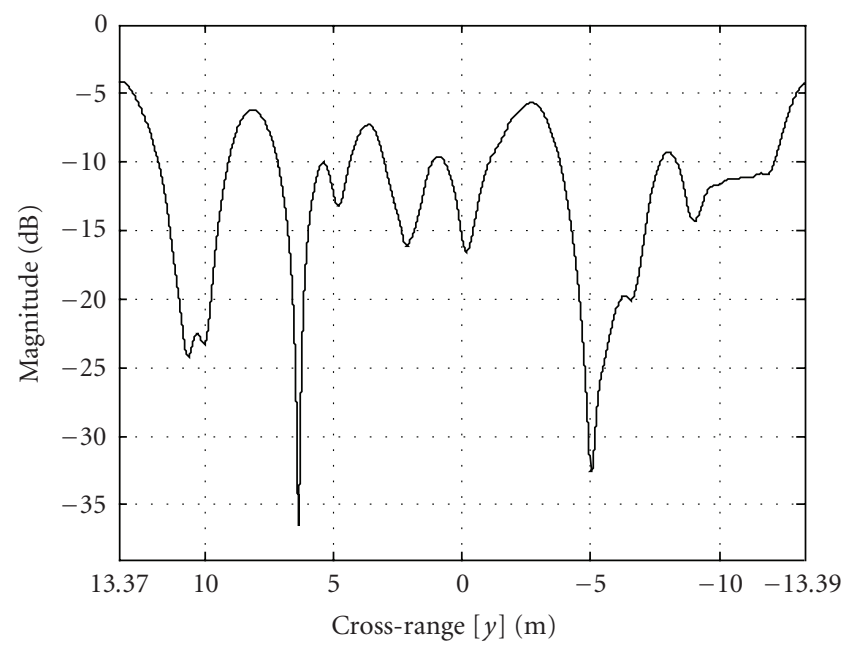

(b)

FIGURE 17: Azimuth cross-sections taken along the building at $240 \mathrm{~m}$ for the (a) moving heterodyne and (b) stationary heterodyne images.

An imaging algorithm for SS-BSAR was briefly discussed and experimentally tested. Using the flight imitator, bistatic images were successfully obtained for moving and stationary heterodyne channel. These images were also briefly analysed. Comparison of moving heterodyne image with that of stationary heterodyne channel showed high level of similarity, suggesting our new (moving heterodyne) configuration is functioning properly. Finally, we can conclude that we can obtain an SS-BSAR image for a moving heterodyne channel. The next stage of our work will concentrate on obtaining image using a real aircraft.

\section{ACKNOWLEDGMENT}

The work reported in this paper was funded by the ElectroMagnetic Remote Sensing (EMRS) Defence Technology Centre, established by the UK Ministry of Defence and run 
by a consortium of SELEX Sensors and Airborne Systems, Thales Defence, Roke Manor Research, and Filtronic. Project no. $1 / 27$.

\section{REFERENCES}

[1] P. Dubois-Fernandez, H. Cantalloube, B. Vaizan, et al., "ONERA-DLR bistatic SAR campaign: planning, data acquisition, and first analysis of bistatic scattering behaviour of natural and urban targets," IEE Proceedings: Radar, Sonar and Navigation, vol. 153, no. 3, pp. 214-223, 2006.

[2] I. Walterscheid, J. H. G. Ender, A. R. Brenner, and O. Loffeld, "Bistatic SAR processing and experiments," IEEE Transactions on Geoscience and Remote Sensing, vol. 44, no. 10, pp. 27102717, 2006.

[3] A. Moccia, N. Chiacchio, and A. Capone, "Spaceborne bistatic synthetic aperture radar for remote sensing applications," International Journal of Remote Sensing, vol. 21, no. 18, pp. 3395-3414, 2000.

[4] M. Younis, R. Metzig, and G. Krieger, "Performance prediction of a phase synchronization link for bistatic SAR," IEEE Geoscience and Remote Sensing Letters, vol. 3, no. 3, pp. 429433, 2006.

[5] M. Cherniakov, "Space-surface bistatic synthetic aperture radar-prospective and problems," in Proceedings of the IEEE International Radar Conference, no. 490, pp. 22-25, Edinburgh, UK, October 2002.

[6] http://www.en.wilkipedia.org/wiki/GPS_modernization.

[7] M. Cherniakov, R. Saini, M. Antoniou, R. Zuo, and J. Edwards, "SS-BSAR with transmitter of opportunitypractical aspects," in Proceedings of the 3rd EMRS DTC Technical Conference, Edinburgh, UK, July 2006.

[8] X. He, M. Cherniakov, and T. Zeng, "Signal detectability in SS-BSAR with GNSS non-cooperative transmitter," IEE Proceedings: Radar, Sonar and Navigation, vol. 152, no. 3, pp. 124-132, 2005.

[9] X. He, T. Zeng, and M. Cherniakov, "Interference level evaluation in SS-BSAR with GNSS non-cooperative transmitter," Electronics Letters, vol. 40, no. 19, pp. 1222-1224, 2004.

[10] U. Roßbach, Positioning and navigation using the Russian satellite system GLONASS, Ph.D. thesis, Universität der Bundeswehr München, Munchen, Germany, 2000.

[11] J. B.-Y. Tsui, Fundamentals of Global Positioning System Receivers: A Software Approach, John Wiley \& Sons, New York, NY, USA, 2000.

[12] M. Antoniou, Image formation algorithms for space-surface bistatic SAR, Ph.D. thesis, University of Birmingham, Birmingham, UK, 2007.

[13] M. Antoniou, M. Cherniakov, and C. Hu, "Space-surface BSAR image formation algorithms," submitted to IEEE Transactions in Geoscience and Remote Sensing.

[14] M. Cherniakov, M. Antoniou, R. Saini, R. Zuo, and J. Edwards, "Space-surface BSAR - analytical and experimental study," in Proceedings of the 6th European Conference on Synthetic Aperture Radar (EUSAR '06), Dresden, Germany, May 2006.

[15] M. Antoniou, R. Saini, and M. Cherniakov, "Results of a space-surface bistatic SAR image formation algorithm," IEEE Transactions on Geoscience and Remote Sensing, vol. 45, no. 11, pp. 3359-3371, 2007.

[16] M. Antoniou, R. Saini, R. Zuo, and M. Cherniakov, "Image formation algorithm for space-surface BSAR," in Proceedings of the 4th European Radar Conference (EuRAD '07), pp. 413416, Munich, Germany, October 2007.
[17] M. Antoniou, R. Saini, R. Zuo, and M. Cherniakov, "Spacesurface bistatic SAR topology and its impact on image formation," in Proceedings of the 7th European Conference on Synthetic Aperture Radar (EUSAR '08), Friedrichshafen, Germany, June 2008.

[18] I. G. Cumming and F. H. Wong, Digital Processing of Synthetic Aperture Radar Data: Algorithms and Implementation, Artech House, Norwood, Mass, USA, 2005.

[19] R. Zuo, Test bed development for space-surface bistatic SAR investigation, M.S. thesis, University of Birmingham, Birmingham, UK, October 2005.

[20] M. Cherniakov, R. Saini, R. Zuo, and M. Antoniou, "Spacesurface bistatic synthetic aperture radar with global navigation satellite system transmitter of opportunity-experimental results," IET Radar, Sonar \& Navigation, vol. 1, no. 6, pp. 447458, 2007. 

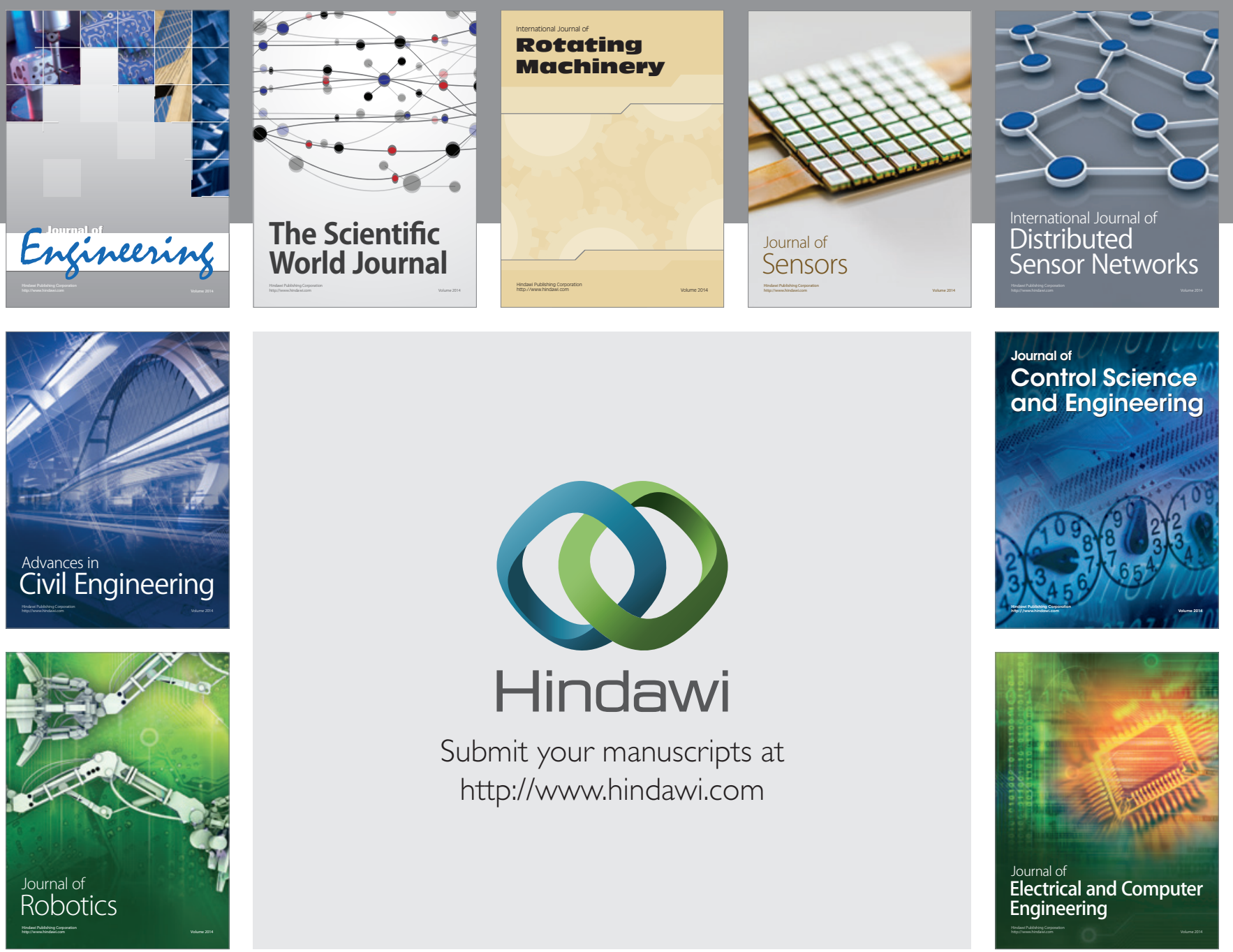

Submit your manuscripts at

http://www.hindawi.com
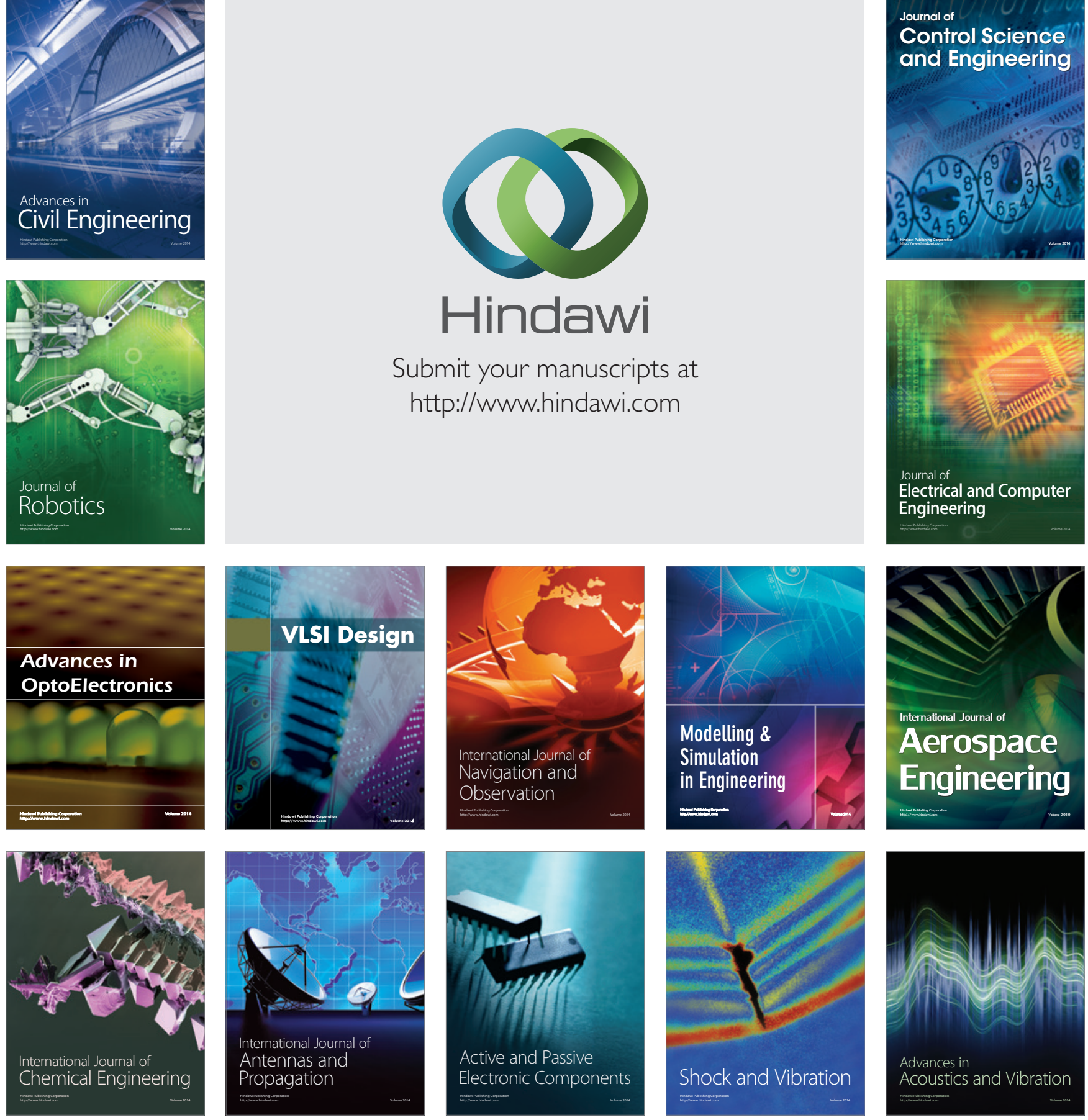